\title{
Distinction of endometrial stromal sarcomas from 'hemangiopericytomatous' tumors using a panel of immunohistochemical stains
}

\author{
Rohit Bhargava ${ }^{1}$, Jinru Shia ${ }^{1}$, Amanda J Hummer ${ }^{2}$, Howard T Thaler ${ }^{2}$, Carmen Tornos ${ }^{1}$ \\ and Robert A Soslow ${ }^{1}$ \\ ${ }^{1}$ Department of Pathology and ${ }^{2}$ Department of Epidemiology and Biostatistics, Memorial Sloan-Kettering \\ Cancer Center, New York, NY, USA
}

\begin{abstract}
Endometrial stromal sarcomas are low-grade malignant tumors that may pose a diagnostic challenge, especially when they are present in an extrauterine site. Owing to the presence of an arborizing vasculature and cells with an undifferentiated appearance, endometrial stromal sarcomas can be confused with several softtissue neoplasms. We studied 17 endometrial stromal sarcomas, eight hemangiopericytomas, 14 solitary fibrous tumors, and 16 synovial sarcomas immunohistochemically, detecting the following antigens: CD10, estrogen receptor, progesterone receptor, bcl-2, CD34, smooth muscle antigen, epithelial membrane antigen and cytokeratin (AE1/AE3). Most endometrial stromal sarcomas stained positively for CD10 (16/17), estrogen receptor (17/17), progesterone receptor (15/17), and bcl-2 (17/17). Staining with antismooth muscle antigen was seen in 11 of 17 cases of endometrial stromal sarcoma, with more intense staining seen in areas showing smooth muscle differentiation. Staining with $A E 1 / 3$ was seen in four of 17 endometrial stromal sarcomas, with two of the positive cases containing epithelioid cells. None of the endometrial stromal sarcomas expressed epithelial membrane antigen or CD34. More than half of the hemangiopericytomas (4/8) and solitary fibrous tumors (9/14) cases demonstrated CD10 expression either focally or in a patchy cytoplasmic and membranous pattern. Hemangiopericytomas, solitary fibrous tumors, and synovial sarcomas did not express estrogen receptor. Four of eight hemangiopericytomas and seven of 14 solitary fibrous tumors also showed patchy progesterone receptor expression. CD34 expression was identified in six of eight hemangiopericytomas and 13 of 14 solitary fibrous tumors, but we did not find expression of CD34 in synovial sarcoma. Differences between endometrial stromal sarcoma and other soft-tissue tumors were detected for all of the immunohistochemical markers $(P<0.05)$, except anti-bcl-2 and AE1/3. Antibodies against CD10 mark a substantial number of hemangiopericytomas and solitary fibrous tumors (albeit not diffusely) and should always be combined with antiestrogen receptor and CD34 when the differential diagnosis includes endometrial stromal sarcoma. Unlike estrogen receptor antibodies, progesterone receptor antibodies show at least focal nuclear staining in most hemangiopericytomas, solitary fibrous tumors and rare synovial sarcomas, and are not useful for this differential diagnosis. All endometrial stromal sarcomas expressed bcl-2, mostly in a diffuse pattern, but this did not distinguish between endometrial stromal sarcoma and mimics. We therefore recommend the use of a small antibody panel comprising anti-CD10, anti-estrogen receptor, and anti-CD34 to distinguish endometrial stromal sarcomas from tumors with a predominant hemangiopericytomatous growth pattern.
\end{abstract}

Modern Pathology (2005) 18, 40-47, advance online publication, 13 August 2004; doi:10.1038/modpathol.3800248

Keywords: differential diagnosis; endometrial stromal sarcoma; hemangiopericytoma; immunohistochemistry; solitary fibrous tumor; synovial sarcoma

Endometrial stromal tumors have been traditionally classified into endometrial stromal nodule and endometrial stromal sarcoma. ${ }^{1}$ Endometrial stromal

Correspondence: Dr RA Soslow, MD, Department of Pathology, Memorial Sloan-Kettering Cancer Center, 1275 York Avenue, New York, NY 10021, USA.

E-mail: soslowr@mskcc.org

Received 24 March 2004; revised 22 June 2004; accepted 23 June 2004; published online 13 August 2004 sarcoma comprises $<10 \%$ of the uterine mesenchymal neoplasms, and approximately one-half of women affected are premenopausal. ${ }^{2}$ Endometrial stromal sarcomas are indolent tumors with local recurrences and distant metastasis occurring even 20 years after initial diagnosis. ${ }^{3}$ Several morphologic variants of endometrial stromal sarcoma have been described; they include those with fibromyxoid features and those showing smooth muscle, fibroblastic, and epithelial differentiation, which 
sometimes resembles endometrial stromal sarcoma with sex cord elements. ${ }^{4-7}$ One unifying feature encountered in most cases is the presence of a prominent arborizing and pericytomatous vasculature. Because of this feature, endometrial stromal sarcoma may be confused with hemangiopericytoma and other soft-tissue tumors. It is now generally accepted that most uterine hemangiopericytomas reported in the literature probably represented examples of endometrial stromal tumors. ${ }^{8-10}$ With late recurrences and metastasis in unusual locations and varied histology, the differential diagnosis of metastatic endometrial stromal sarcoma is wide and includes several soft-tissue neoplasms demonstrating arborizing vasculature. With the intent of distinguishing endometrial stromal sarcoma from soft-tissue neoplasms demonstrating 'hemangiopericytomatous' vascular patterns, we studied 55 cases of endometrial stromal sarcoma, solitary fibrous tumor, hemangiopericytoma, and synovial sarcoma using a battery of commonly used immunohistochemical stains-anti-CD10, anti-estrogen receptor (ER), anti-progesterone receptor (PR), anti-bcl-2, anti-CD34, anti-smooth muscle antigen (SMA), anti-epithelial membrane antigen (EMA), and AE1/ AE3 cytokeratin.

\section{Materials and methods}

In all, 17 low-grade endometrial stromal sarcoma cases (eight with classic histology and nine with variant histology) were retrieved from the surgical pathology files at Memorial Sloan-Kettering Cancer Center. The nine variant endometrial stromal sarco- ma tumors included five with smooth muscle differentiation, and one each with fibroblastic differentiation, fibromyxoid features, epithelioid cellular elements, and with sex cord differentiation. In total, 38 soft-tissue tumors, including eight hemangiopericytomas, 14 solitary fibrous tumors, and 16 synovial sarcomas, were also retrieved from the surgical pathology files at our institution. The clinical data for the patient population are provided in Table 1. The hemangiopericytoma and solitary fibrous tumor cases selected for study had ultrastructure examination performed at presentation to confirm the diagnosis. We used the presence of basal lamina material in hemangiopericytomas to separate them from solitary fibrous tumors. They were otherwise very similar from a morphologic standpoint. All synovial sarcoma cases had molecular evidence of $t(X ; 18)$ translocation by reverse transcription-polymerase chain reaction. One representative formalin-fixed paraffin-embedded tissue block was selected from each case and submitted for immunohistochemical study. The immunohistochemical stains were performed with the biotinstreptavidin complex technique using the primary antibodies detailed in Table 2. Appropriate positive and negative controls were performed.

\section{Statistical Analysis}

$\chi^{2}$-tests were performed to determine if there were any immunohistochemical differences between endometrial stromal sarcoma and soft-tissue tumors included in this study. In order to determine which combination of the eight markers best distinguished

Table 1 Clinical data

\begin{tabular}{lccl}
\hline & $\begin{array}{c}\text { Mean age in } \\
\text { years (range) }\end{array}$ & Sex & Tumor location (number of cases) \\
\hline ESS & $47(21-72)$ & All F & Uterus (8), abdominal wall (3), lung (3), retroperitoneum (2), pelvis (1) \\
HPC & $47(30-72)$ & $5 \mathrm{M} / 3 \mathrm{~F}$ & Pelvis (2), extremity (2), abdominal wall (1), ischium soft tissue (1), lung/pleura (1), head and neck (1) \\
SFT & $62(40-82)$ & $8 \mathrm{M} / 6 \mathrm{~F}$ & Lung/pleura (7), thorax/mediastinum (2), retroperitoneum (2), extremity (2), head and neck (1) \\
SS & $36(16-67)$ & $8 \mathrm{M} / 8 \mathrm{~F}$ & Extremity (8), lung/pleura (2), axilla (2), retroperitoneum (1), mediastinum (1), head and neck (1)
\end{tabular}

ESS, endometrial stromal sarcoma; HPC, hemangiopericytoma; SFT, solitary fibrous tumor; SS, synovial sarcoma; M, male; F, female.

Table 2 Detail of antibodies used in the current study

\begin{tabular}{|c|c|c|c|}
\hline Antigen/antibody & Source & Pretreatment & Dilution \\
\hline CD10 (M) & Novocastra Laboratories, Newcastle, UK & Citric acid, $\mathrm{pH}=6$; heat $\left(30 \mathrm{~min}, 98^{\circ} \mathrm{C}\right)$ & $1: 100$ \\
\hline ER (M), prediluted & Immunotech, Marseille, France & Same & $1: 100$ \\
\hline PR (M) & Biogenex, San Ramon, CA, USA & Same & $1: 200$ \\
\hline BCL2 (M) & Dako Corporation, Carpinteria, CA, USA & Same & $1: 50$ \\
\hline CD34 (M) & Immunotech, Marseille, France & Same & $1: 4000$ \\
\hline SMA (M) & Dako Corporation, Carpinteria, CA, USA & Same & 1:1000 \\
\hline EMA (M) & Dako Corporation, Carpinteria, CA, USA & None & $1: 100$ \\
\hline $\mathrm{AE} 1 / 3(\mathrm{M})$ & Biogenex, San Ramon, CA, USA & Protease, $10 \mathrm{~min}$ & $1: 600$ \\
\hline
\end{tabular}

M, monoclonal; ER, estrogen receptor; PR, progesterone receptor; SMA, smooth muscle antigen; EMA, epithelial membrane antigen. 
endometrial stromal sarcomas from the soft-tissue tumors, we used stepwise discriminant analysis. Variables entered into a multivariate model had a univariate $P$-value of 0.05 . To determine the strength of the multivariate model, a logistic regression analysis was performed using the variables that remained significant from the multivariate discriminant analysis. From this, we obtained a model $R^{2}$ value. Statistical analysis were performed using SAS Software (SAS Institute, Inc., Cary, NC, USA).

\section{Results}

The number of cases positive with a particular antibody/total number of cases in each diagnostic category with percentage positivity in parentheses is shown in Table 3. Following is a description of the staining patterns of the various markers in each diagnostic category.

\section{Endometrial Stromal Sarcoma}

\section{CD10}

A total of $16(94 \%)$ of 17 endometrial stromal sarcomas were positive with the CD10 antibody. Of the eight cases showing classic (nonvariant) histology, four demonstrated diffuse strong staining (Figure 1a and b), two showed patchy cytoplasmic and membranous staining, and two were only focally positive. Of the five cases of endometrial stromal sarcoma with smooth muscle differentiation, three showed patchy cytoplasmic and membranous staining pattern, one was diffusely positive, and one was negative. One fibromyxoid variant and one case showing epithelioid differentiation showed diffuse positivity. One case of endometrial stromal sarcoma with sex cord differentiation showed patchy cytoplasmic and membranous staining and one case of endometrial stromal sarcoma with fibroblastic differentiation was only focally positive.

\section{ER}

All cases (17/17) of endometrial stromal sarcoma, including the variants, showed nuclear ER expres- sion (Figure 1c), although three cases with classic histology showed only focal nuclear staining.

\section{$P R$}

Totally, 15 (88\%) of 17 cases were positive for the PR antibody (Figure 1d). Two of 15 positive cases with classic histology showed focal and patchy nuclear staining. Two negative cases included one endometrial stromal sarcoma with smooth muscle differentiation and one with fibroblastic differentiation.

\section{bcl-2}

All cases (17/17) of endometrial stromal sarcoma were positive with anti-bcl-2 showing predominantly a diffuse cytoplasmic and membranous staining. Focal staining was observed in two endometrial stromal sarcomas with smooth muscle differentiation and one with fibroblastic differentiation.

\section{CD34}

All cases (17/17) were negative for CD34.

\section{$S M A$}

Overall, 11 (65\%) of 17 cases were positive with the SMA antibody. All endometrial stromal sarcomas with smooth muscle differentiation (5/5) showed diffuse positivity. Of the eight endometrial stromal sarcomas with classic histology, five were negative and three were positive, of which, two showed only focal staining. One endometrial stromal sarcoma with fibroblastic differentiation and one with epithelioid features were also positive. Endometrial stromal sarcoma with sex cord differentiation showed focal positivity. One endometrial stromal sarcoma with fibromyxoid features was negative for SMA.

\section{$E M A$}

All cases (17/17) were negative with EMA antibodies.

\section{AE1/3}

Endometrial stromal sarcomas showing epithelioid cellular elements and sex cord differentiation were both positive with AE1/3, specifically in epithelioid

Table 3 Staining of all cases in each diagnostic category

\begin{tabular}{|c|c|c|c|c|c|c|c|c|}
\hline & CD10 (\%) & $E R(\%)$ & $P R(\%)$ & $b c l-2(\%)$ & CD34 (\%) & $S M A(\%)$ & $E M A(\%)$ & $A E 1 / 3(\%)$ \\
\hline ESS & $16 / 17^{\mathrm{a}}(94)$ & $17 / 17(100)$ & 15/17 (88) & $17 / 17(100)$ & 0/17 (0) & $11 / 17(65)$ & $0 / 17(0)$ & $4 / 17(24)$ \\
\hline HPC & $4 / 8^{a}(50)$ & 0/8 (0) & $4 / 8(50)$ & 8/8 (100) & 6/8 (75) & $2 / 8(25)$ & 1/8 (13) & $3 / 8(38)$ \\
\hline SFT & $9 / 14^{\mathrm{a}}(64)$ & $0 / 14(0)$ & $7 / 14(50)$ & 13/14 (93) & 13/14 (93) & $3 / 14(21)$ & $0 / 14(0)$ & $0 / 14(0)$ \\
\hline SS & $1 / 16(6)$ & 0/16 (0) & $1 / 16(6)$ & $16 / 16(100)$ & $0 / 16(0)$ & $2 / 16(13)$ & $12 / 16(75)$ & $13 / 16(81)$ \\
\hline$P$-value ${ }^{\mathrm{b}}$ & 0.004 & $<0.001$ & $<0.001$ & 0.51 & $<0.001$ & $<0.001$ & 0.005 & 0.19 \\
\hline
\end{tabular}

ER, estrogen receptor; PR, progesterone receptor; SMA, smooth muscle antigen; EMA, epithelial membrane antigen; ESS, endometrial stromal sarcoma; HPC, hemangiopericytoma; SFT, solitary fibrous tumor; SS, synovial sarcoma.

${ }^{\mathrm{a}}$ Patchy cytoplasmic and membranous staining pattern was observed in HPC, SFT, and ESS, but diffuse staining was seen only in ESS.

${ }^{\mathrm{b}}$ The univariate $P$-values were obtained from $\chi^{2}$-test, which was used to determine if there was difference in positivity for immunohistochemical markers between ESS and soft-tissue tumors. 

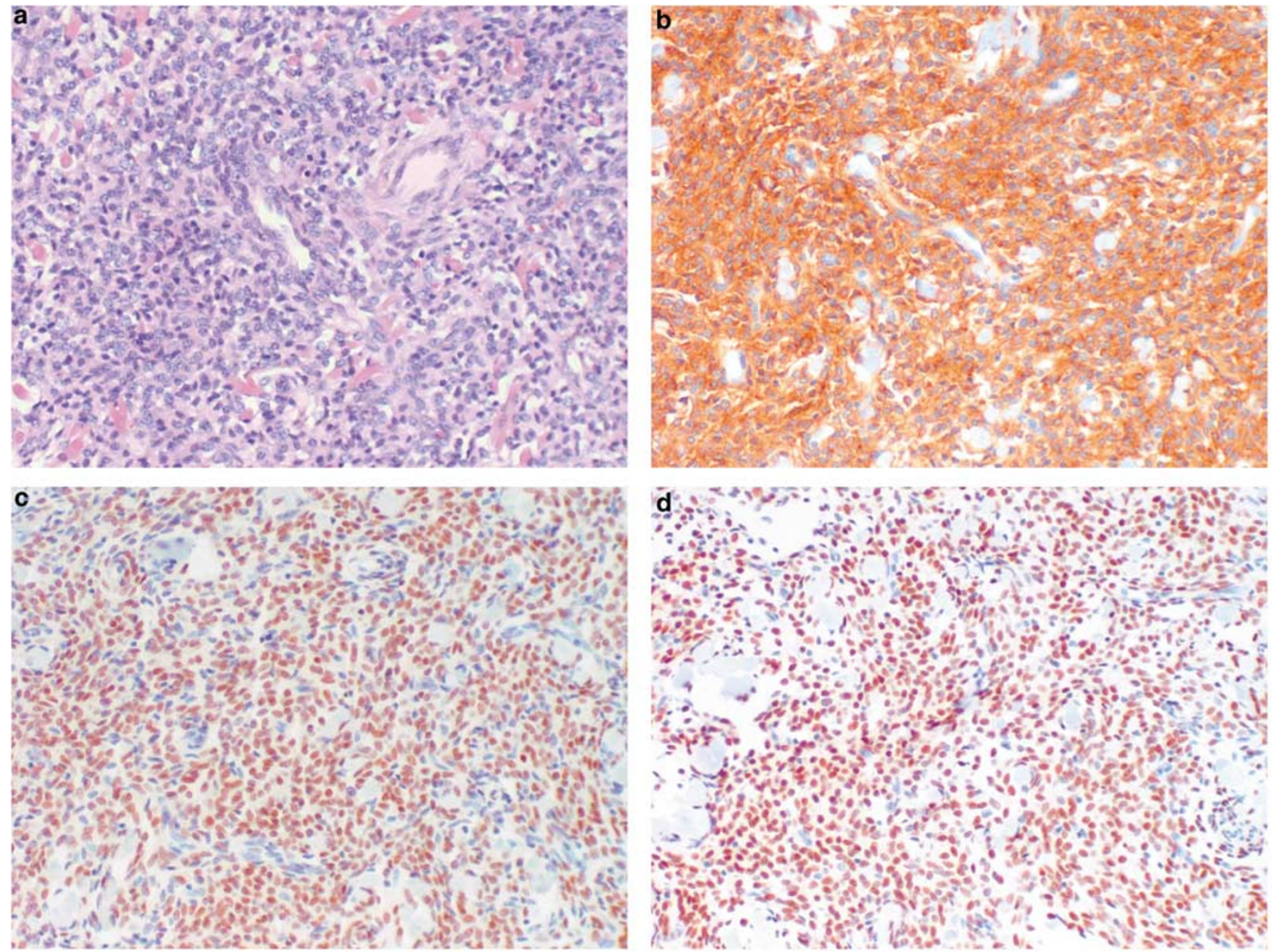

Figure 1 An endometrial stromal sarcoma (a) demonstrating diffuse positivity for CD10 (b), estrogen receptor (c), and progesterone receptor (d).

areas. Two additional cases with classic (nonvariant) histology also showed some positivity. The remainder (13/17) of the endometrial stromal sarcomas were negative.

The immunophenotypes of uterine $(n=8)$ and extrauterine endometrial stromal sarcoma $(n=9)$ were compared. These were roughly similar; no statistically significant differences were observed.

\section{Hemangiopericytoma}

Fifty percent of the cases (4/8) stained with antiCD10. Two of the positive cases showed a patchy cytoplasmic and membranous pattern (Figures 2a and $\mathrm{b}$ ) and the 2 others demonstrated focal positivity with CD10. None showed diffuse strong staining. None of the cases (0/8) showed ER expression; however, $4(50 \%)$ of 8 cases showed patchy nuclear staining with anti-PR. All cases (8/8) were diffusely positive for anti-bcl-2. Six (75\%) of 8 cases were positive for anti-CD34, with 1 case showing only focal positivity. Focal positivity was observed in 3 cases with AE1/3, 2 cases with anti-SMA, and only 1 case with anti-EMA.

\section{Solitary Fibrous Tumor}

In all, $64 \%$ of the cases (9/14) showed staining with the CD10 antibody. Six of the positive cases showed a patchy cytoplasmic and membranous pattern (Figure 2c and d), while three cases demonstrated focal positivity. None showed diffuse strong staining. None of the cases $(0 / 14)$ were positive with antiER; however, seven (50\%) of 14 cases showed patchy nuclear staining with PR antibodies. Totally, $13(93 \%)$ of 14 cases were diffusely positive with anti-bcl-2 and CD34. None of the cases (0/14) stained with anti-EMA or AE1/3. Only three (21\%) of 14 cases showed focal positivity with SMA antibodies.

\section{Synovial Sarcoma}

Only one case showed focal positivity with antiCD10. All cases were negative with ER antibodies. 

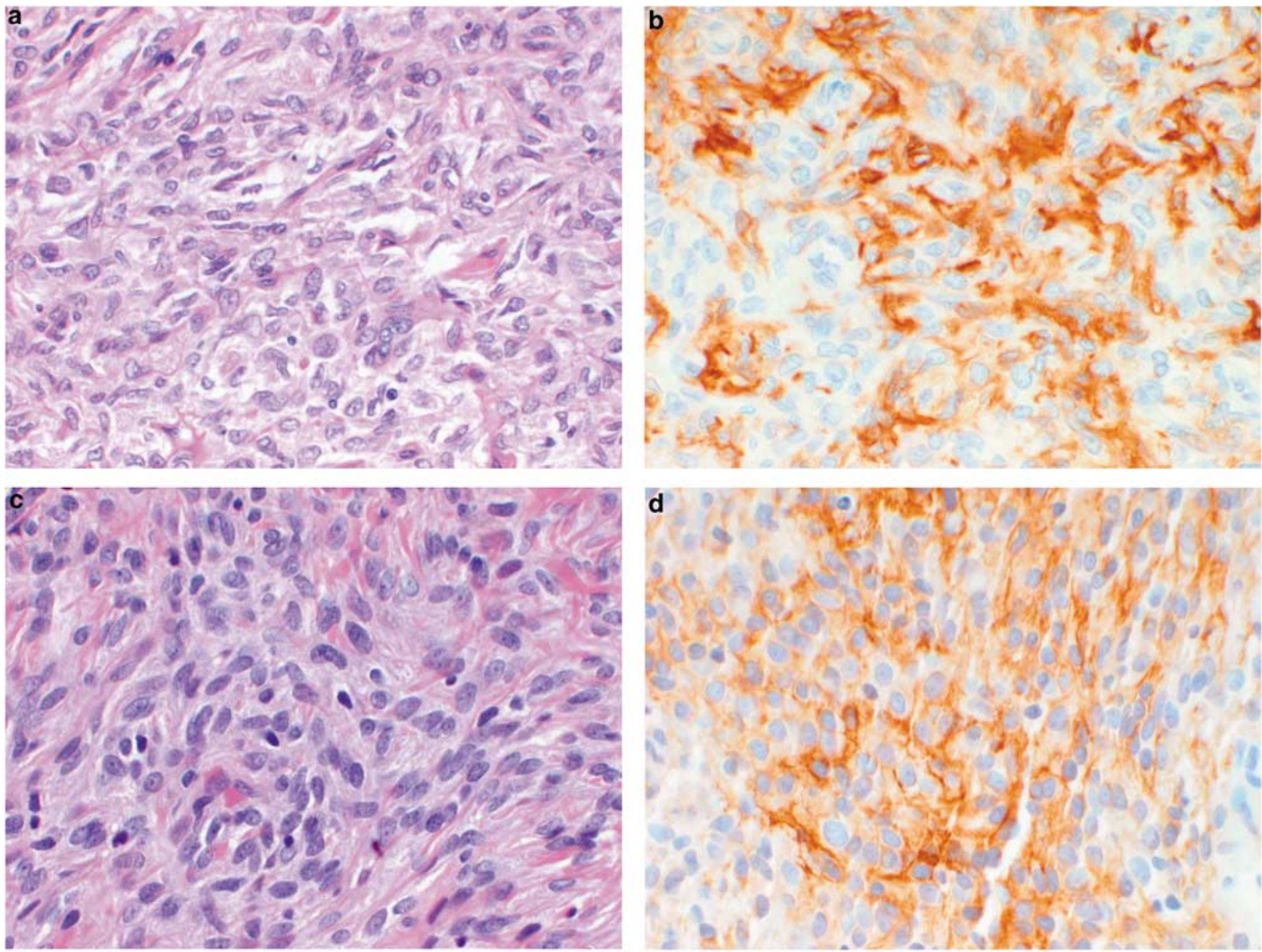

Figure 2 Patchy cytoplasmic and membranous staining for CD10 in a case of hemangiopericytoma (a and b) and solitary fibrous tumor (c and $\mathbf{d}$ ).

Only one case showed patchy and focal nuclear positivity with anti-PR. All cases (16/16) were positive for anti-bcl-2, predominantly in a diffuse pattern. A large majority of the cases were positive with anti-EMA (12/16; 75\%) and AE1/3 (13/16; $81 \%$ ). Two cases demonstrated focal positivity with SMA antibodies. None of the cases $(0 / 16)$ stained with CD34 antibody.

Nine synovial sarcomas were biphasic and seven were monophasic. Significant differences in smooth muscle actin, bcl-2, CD34, PR, ER, and CD10 expression were not noted, but differences were detected for AE1/AE3 and EMA. Four of seven monophasic synovial sarcomas expressed AE1/AE3 cytokeratin compared with nine of nine biphasic tumors. Three of seven monophasic tumors expressed EMA compared with nine of nine biphasic tumors.

\section{Statistical Analysis}

To expand the above data, we wanted to determine whether ESS cases resembled the soft-tissue tumors included in this study. $\chi^{2}$-tests and discriminant analysis were utilized for this purpose. The results of the $\chi^{2}$-tests are shown in Table 3.

With the exception of bcl-2 and AE1/3, univariate discriminant analysis showed a difference in immunohistochemical positivity between endometrial stromal sarcoma and other soft-tissue tumors $(P$ value $<0.05)$. Those markers with a $P$-value $<0.05$ were then included in a stepwise multivariate discriminant analysis.

Using stepwise discriminant analysis, we could determine which combination of immunohistochemical markers best discriminated endometrial stromal sarcoma from soft-tissue tumors. When including all markers with a univariate $P$-value $<0.05$ in a multivariate analysis, ER was the only marker that remained significant. ER had perfect discrimination and accounted for the other five markers' effect in the model (partial $R^{2}: 1.00, P$-value $<0.001$, model $\left.R^{2}: 0.71\right)$. We then excluded markers' with univariate $P$-values $>0.05$ and ER and found the combination of positive CD10, positive PR, negative CD34 and negative EMA distinguished 
ESS from other soft-tissue tumors. Using ER antibodies along with those against CD10 and CD34 would account for all the important entities in the differential diagnosis. This panel is therefore preferable to immunostaining for ER alone.

\section{Discussion}

Endometrial stromal sarcomas are rare uterine mesenchymal neoplasms. Diagnostic features include their resemblance to normal proliferative phase endometrium, lack of significant cytologic atypia, and presence of infiltrative borders with or without vascular space invasion. It can be difficult to establish an endometrial stromal sarcoma diagnosis since they often display protean appearances, may arise as primary neoplasms outside the uterus, and metastasize decades after hysterectomy. A prominent pericytomatous vascular pattern can cause confusion with solitary fibrous tumor, hemangiopericytoma, and synovial sarcoma. Although electron microscopy and molecular techniques can be used in making a definitive diagnosis, these techniques are expensive and are not always available. On the contrary, immunohistochemical analysis is much less expensive and is readily available at most institutions. We carried out this immunohistochemical study with some commonly used antibodies in order to distinguish endometrial stromal sarcomas from several soft tissue neoplasms with hemangiopericytomatous vascular pattern.

CD10 is a cell surface neutral endopeptidase, characterized originally on immature lymphoid cells. ${ }^{11-13}$ With the advent of immunohistochemistry, it was found to be expressed in renal tubular and glomerular cells, breast and salivary gland myoepithelial cells, and prostatic glandular and pulmonary alveolar lining cells. ${ }^{14-18}$ Recently, CD10 expression has been shown in several nonhematopoietic neoplasms, including endometrial stromal sarcomas. ${ }^{19}$ Furthermore, CD10 was identified as a sensitive and diagnostically useful marker of normal endometrial stroma and of endometrial stromal neoplasms. ${ }^{20-23}$ In this study, antibodies against CD10 stained a majority of endometrial stromal sarcomas in a diffuse pattern, but in some cases the staining pattern was focal or patchy cytoplasmic and membranous. A similar patchy cytoplasmic and membranous pattern of staining was also observed in many solitary fibrous tumors and hemangiopericytomas. Thus, only strong diffuse staining, seen in seven of 17 endometrial stromal sarcomas, was specific for this diagnosis. Patchy cytoplasmic and membranous staining as well as focal staining was more prevalent in variant tumors than in classical (nonvariant) endometrial stromal sarcomas.

Immunohistochemical staining with hormone receptors was variable. ER staining showed expected results in most endometrial stromal sarcomas, with diffuse nuclear positivity, and a lack of
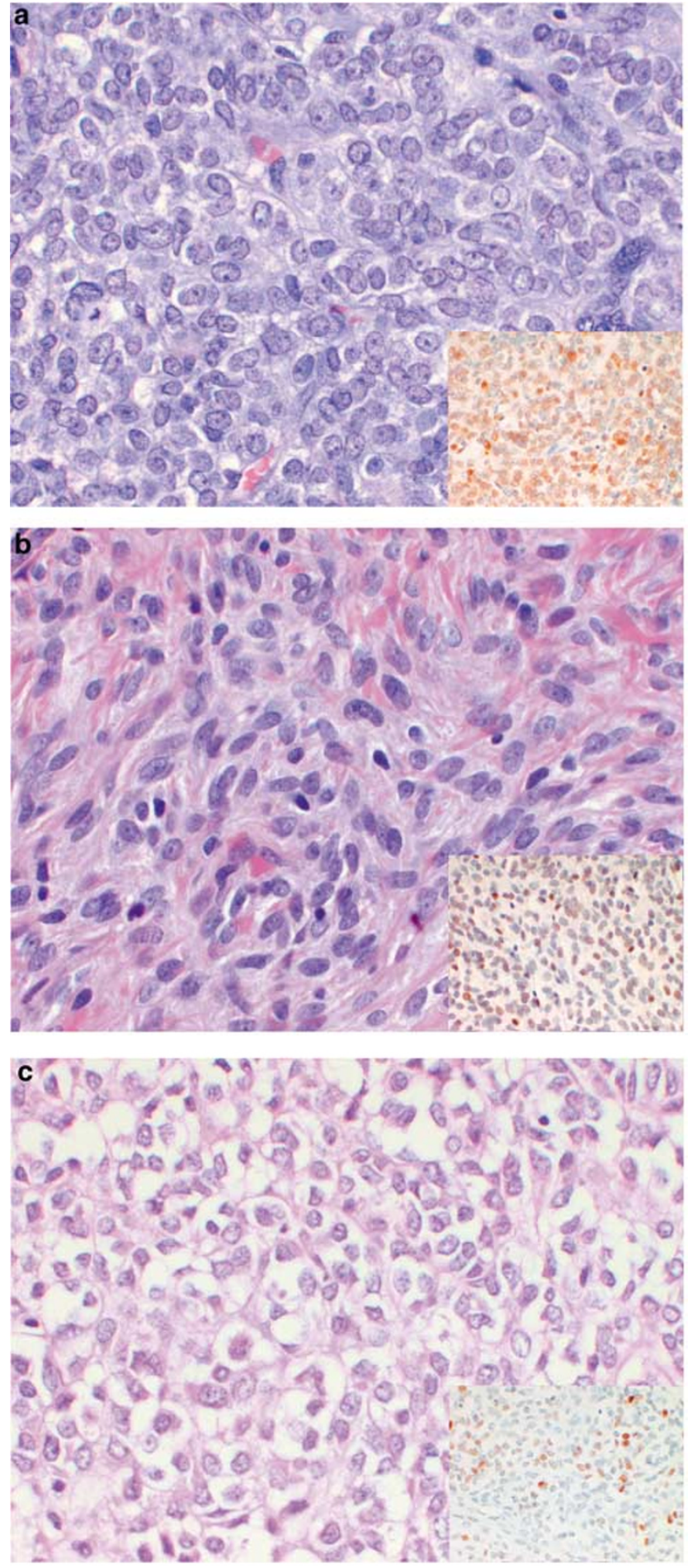

Figure 3 Patchy nuclear staining with PR (inset) in hemangiopericytoma (a), solitary fibrous tumor (b), and synovial sarcoma (c).

staining in solitary fibrous tumor, hemangiopericytoma, and synovial sarcoma. On the contrary, PR staining revealed some surprising results. Endometrial stromal sarcomas showed a high rate of positivity (ranging from focal patchy to diffuse), but $50 \%$ of the hemangiopericytomas and solitary 
fibrous tumors also showed patchy PR expression (Figure 3a and b). One synovial sarcoma case also showed rare PR-positive cells (Figure 3c). There are only rare studies that have reported PR expression in solitary fibrous tumors. ${ }^{24,25}$ The significance of this is not known, but it is important to recognize this diagnostic pitfall.

bcl-2 expression has been previously reported in proliferative phase and late secretory phase endometrial stroma, ectopic endometrium, and adenomyosis but has never been reported in endometrial stromal sarcoma, to our knowledge. ${ }^{26-29}$ All of the endometrial stromal sarcomas in this study were diffusely positive with anti-bcl-2, which may suggest an etiologic role for this antiapoptotic gene in tumor progression. However, since solitary fibrous tumors, hemangiopericytomas, and synovial sarcomas also stained with bcl-2, we concluded that bcl-2 was not useful in this differential diagnosis.

The CD34 antibody stained only the solitary fibrous tumors and hemangiopericytomas and therefore can be used to distinguish these from endometrial stromal sarcoma, as none of the endometrial stromal sarcomas stained with this antibody.

Among endometrial stromal sarcomas, SMA was expressed predominantly in areas showing smooth muscle differentiation; focal staining was observed in classical (nonvariant) endometrial stromal areas as well. Hemangiopericytomas and solitary fibrous tumors, also expressed SMA, which has been reported previously. ${ }^{30}$ Although not used in this study, more specific smooth muscle markers, such as desmin and especially h-caldesmon, can be used to confirm smooth muscle differentiation.

None of the endometrial stromal sarcomas were positive with EMA antibodies, which is in accordance with a previous report. ${ }^{31}$ EMA expression was mainly observed in glandular components of biphasic synovial sarcomas, as expected.

AE1/3 staining was observed in endometrial stromal sarcoma cases showing epithelial and sex cord stromal differentiation. The case with sex cord stromal differentiation also showed anti-SMA staining, consistent with an epithelial-myoid phenotype of some sex cord elements in endometrial stromal sarcoma. ${ }^{22,32,33}$ This case did not express EMA. Two other cases of endometrial stromal sarcoma with classic (nonvariant) histology showed some AE1/3 positivity, as previously reported ${ }^{30}$ but the significance of this finding is not known.

Therefore, it appears that evaluation for CD10 immunoreactivity alone is frequently not informative about differentiating endometrial stromal sarcomas from mimics with pericytomatous vascular patterns. The statistical analysis suggests that although ER best discriminates between entities there is a panel of markers that also discriminates well. A panel of immunohistochemical stains comprising anti-CD10, anti-ER, and anti-CD34 may be more helpful. PR evaluation may have therapeutic relevance in endometrial stromal sarcoma, but the results are not as useful in distinguishing endometrial stromal sarcoma from solitary fibrous tumor or hemangiopericytoma. We also confirm that endometrial stromal sarcoma variants can lose CD10 immunoreactivity and may stain with epithelial and myoid markers depending on differentiation.

\section{Acknowledgements}

We thank Irina Linkov and Marina Asherova for excellent technical support.

\section{References}

1 Norris HJ, Taylor HB. Mesenchymal tumors of the uterus. I. A clinical and pathological study of 53 endometrial stromal tumors. Cancer 1966;19:755-766.

2 Fekete PS, Vellios F. The clinical and histologic spectrum of endometrial stromal neoplasms: a report of 41 cases. Int J Gynecol Pathol 1984;3:198-212.

3 Inayama Y, Shoji A, Odagiri S, et al. Detection of pulmonary metastasis of low-grade endometrial stromal sarcoma 25 years after hysterectomy. Pathol Res Pract 2000;196:129-134.

4 Oliva E, Clement PB, Young RH, et al. Mixed endometrial stromal and smooth muscle tumors of the uterus: a clinicopathologic study of 15 cases. Am J Surg Pathol 1998;22:997-1005.

5 Oliva E, Young RH, Clement PB, et al. Myxoid and fibrous endometrial stromal tumors of the uterus: a report of 10 cases. Int J Gynecol Pathol 1999;18: 310-319.

6 Oliva E, Clement PB, Young RH. Endometrial stromal tumors: an update on a group of tumors with a protean phenotype. Adv Anat Pathol 2000;7:257-281.

7 Yilmaz A, Rush DS, Soslow RA. Endometrial stromal sarcomas with unusual histologic features: a report of 24 primary and metastatic tumors emphasizing fibroblastic and smooth muscle differentiation. Am J Surg Pathol 2002;26:1142-1150.

8 Betta PG, Robutti F. Hemangiopericytoma of the uterus vs. hemangiopericytoma-like endometrial stromal sarcoma: a histopathological approach to differential diagnosis. Eur J Obstet Gynecol Reprod Biol 1985;20:235-240.

9 Munoz AK, Berek JS, Fu YS, et al. Pelvic hemangiopericytomas: a report of five cases and literature review. Gynecol Oncol 1990;36:380-382.

10 Sooriyaarachchi GS, Ramirez G, Roley EL. Hemangiopericytoma of the uterus: report of a case with a comprehensive review of the literature. J Surg Oncol 1978;10:399-406.

11 Greaves MF, Brown G, Rapson NT, et al. Antisera to acute lymphoblastic leukemia cells. Clin Immunol Immunopathol 1975;4:67-84.

12 Greaves MF, Hariri G, Newman RA, et al. Selective expression of the common acute lymphoblastic leukemia (gp 100) antigen on immature lymphoid cells and their malignant counterparts. Blood 1983;61:628-639.

13 Weiss LM, Bindl JM, Picozzi VJ, et al. Lymphoblastic lymphoma: an immunophenotype study of 26 cases with comparison to T cell acute lymphoblastic leukemia. Blood 1986;67:474-478. 
14 Erdos EG, Schulz WW, Gafford JT, et al. Neutral metalloendopeptidase in human male genital tract. Comparison to angiotensin I-converting enzyme. Lab Invest 1985;52:437-447.

15 Gusterson BA, Monaghan $\mathrm{P}$, Mahendran $\mathrm{R}$, et al. Identification of myoepithelial cells in human and rat breasts by anti-common acute lymphoblastic leukemia antigen antibody A12. J Natl Cancer Inst 1986;77:343-349.

16 Johnson AR, Ashton J, Schulz WW, et al. Neutral metalloendopeptidase in human lung tissue and cultured cells. Am Rev Respir Dis 1985;132:564-568.

17 Mahendran R, McIlhinney R, O’Hare M, et al. Expression of the common acute lymphoblastic leukaemia antigen (CALLA) in the human breast. Mol Cell Probes 1989;3:39-44.

18 Metzgar RS, Borowitz MJ, Jones NH, et al. Distribution of common acute lymphoblastic leukemia antigen in nonhematopoietic tissues. J Exp Med 1981;154: 1249-1254.

19 Chu P, Arber DA. Paraffin-section detection of CD10 in 505 nonhematopoietic neoplasms. Frequent expression in renal cell carcinoma and endometrial stromal sarcoma. Am J Clin Pathol 2000;113:374-382.

20 Chu PG, Arber DA, Weiss LM, et al. Utility of CD10 in distinguishing between endometrial stromal sarcoma and uterine smooth muscle tumors: an immunohistochemical comparison of 34 cases. Mod Pathol 2001;14:465-471.

21 McCluggage WG, Sumathi VP, Maxwell P. CD10 is a sensitive and diagnostically useful immunohistochemical marker of normal endometrial stroma and of endometrial stromal neoplasms. Histopathology 2001;39:273-278.

22 Oliva E, Young RH, Amin MB, et al. An immunohistochemical analysis of endometrial stromal and smooth muscle tumors of the uterus: a study of 54 cases emphasizing the importance of using a panel because of overlap in immunoreactivity for individual antibodies. Am J Surg Pathol 2002;26:403-412.
23 Toki T, Shimizu M, Takagi Y, et al. CD10 is a marker for normal and neoplastic endometrial stromal cells. Int J Gynecol Pathol 2002;21:41-47.

24 Bongiovanni M, Viberti L, Pecchioni C, et al. Steroid hormone receptor in pleural solitary fibrous tumours and CD34+ progenitor stromal cells. J Pathol 2002;198:252-257.

25 Fukunaga M. Atypical solitary fibrous tumor of the vulva. Int J Gynecol Pathol 2000;19:164-168.

26 Gompel A, Sabourin JC, Martin A, et al. Bcl-2 expression in normal endometrium during the menstrual cycle. Am J Pathol 1994;144: 1195-1202.

27 Jones RK, Searle RF, Bulmer JN. Apoptosis and bcl-2 expression in normal human endometrium, endometriosis and adenomyosis. Hum Reprod 1998;13: 3496-3502.

28 Mertens HJ, Heineman MJ, Evers JL. The expression of apoptosis-related proteins Bcl-2 and Ki67 in endometrium of ovulatory menstrual cycles. Gynecol Obstet Invest 2002;53:224-230.

29 Vaskivuo TE, Stenback F, Karhumaa P, et al. Apoptosis and apoptosis-related proteins in human endometrium. Mol Cell Endocrinol 2000;165:75-83.

30 Farhood AI, Abrams J. Immunohistochemistry of endometrial stromal sarcoma. Hum Pathol 1991;22: 224-230.

31 Franquemont DW, Frierson Jr HF, Mills SE. An immunohistochemical study of normal endometrial stroma and endometrial stromal neoplasms. Evidence for smooth muscle differentiation. Am J Surg Pathol 1991;15:861-870.

32 Horn LC, Stegner HE. Uterine stromal tumor with ovarian sex cord differentiation. Pathologe 1995;16:421-425.

33 Krishnamurthy S, Jungbluth AA, Busam KJ, et al. Uterine tumors resembling ovarian sex-cord tumors have an immunophenotype consistent with true sexcord differentiation. Am J Surg Pathol 1998;22 1078-1082. 